\title{
One Step Closer to an Ebola Virus Vaccine
}

\author{
Daniel G. Bausch, M.D., M.P.H.\&T.M.
}

Despite the slowing and eventual halting of the spread of Ebola virus disease (EVD) in West Africa, ${ }^{1,2}$ the battle for preventing and managing future outbreaks is still on. This is no time to be complacent. The scale of the outbreak, in which every few days during the early part of the outbreak about the same number of cases accrued as occurred during the entire 3-month outbreak in Gulu, Uganda, in 2000-2001 — previously the largest outbreak on record - prompted us to pull out all the stops, albeit after a slow start. ${ }^{3}$ Vaccines are a key weapon in our armamentarium against EVD. For some years, a number of promising vaccine candidates have been identified, with many more in development. The two leading candidates are vectored vaccines in which the Ebola virus glycoprotein is presented in a replication-incompetent chimpanzee adenovirus 3 (cAd3) or a replication-competent vesicular stomatitis virus (VSV). Both vaccines showed $100 \%$ protection in nonhuman primates at 4 to 5 weeks after single doses were administered and were rushed into phase 1 trials in hopes that the promise of a vaccine that could help stem an epidemic of EVD could be more than theoretical.

Ledgerwood and colleagues now present in the Journal the final results of the phase 1 VRC 207 trial, a nonrandomized, open-label trial of two dose levels of a cAd3-vectored bivalent vaccine against the two most virulent species of ebolavirus, Zaire and Sudan. ${ }^{4}$ They conclude that the vaccine is without significant safety concerns and is immunogenic, inducing strong humoral and cell-mediated responses. Although the results of the trial are indeed promising, questions remain; both immunogenicity and reactogenicity were dose-dependent. The higher dose, which was required to generate the more vigorous immune response, was also associated with systemic symptoms in $70 \%$ of the participants, including one in whom a high fever (temperature, $39.9^{\circ} \mathrm{C}$ ) developed, and with transient leukopenia in $20 \%$. There were no major adverse effects, but the sample size (10 persons at each dose level) is too small to draw firm conclusions in this regard. Of particular concern is that the virus- specific CD8 T-cell response, which may be a key correlate of protection, ${ }^{5}$ was only $20 \%$ in the lower-dose group and $70 \%$ in the higher-dose group. Getting the dose right has relevance not only for ensuring individual protection and minimizing adverse effects, but also for stretching the vaccine supply to the maximum number of doses possible to combat the ongoing outbreak.

Interpretation of the findings of the study by Ledgerwood et al., as with all studies of filovirus vaccines, is hampered by a lack of knowledge regarding the specific correlates of immunity, although, as the authors point out, the immune responses observed in their study involving humans are consistent with those associated with protection in efficacy studies in nonhuman primates. ${ }^{5}$ The matter is further complicated by a lack of standardization of stock viruses ${ }^{6}$ and the fact that, to achieve $100 \%$ mortality in control animals - and thus interpretable results - in studies in nonhuman primates, an extremely high challenge dose of virus (1000 plaque-forming units) is used, which is probably orders of magnitude higher than the inoculum that typically infects a human. Until the correlates of immunity are better understood, it is impossible to say whether the immune response shown at the lower dose in the study by Ledgerwood et al., which caused fewer side effects, is "good enough." Will similar results be observed in West Africa, where malaria is holoendemic and has been associated with diminished immunogenicity with other vectored vaccines? $?^{7}$ At exactly what time point after vaccination is adequate immunity conferred? This is an important question, given the urgency of this type of situation. Will it be necessary to administer a booster with a modified vaccinia Ankara (MVA) vaccine expressing the Ebola glycoprotein, which has been shown to increase the duration of immunity but would considerably complicate delivery? ${ }^{8}$ Results from ongoing phase 1 trials of a monovalent cAd3EBO Zaire vaccine, which may be more immunogenic than a bivalent formation, ${ }^{9}$ as well as of the VSV-vectored vaccine, in various locations in the United States, Europe, and Africa (outside 
the epidemic area for EVD), are informing the answers to these important questions.

Perhaps one of the only silver linings of the EVD crisis that shook West Africa several years ago is that the event pushed therapeutics and vaccines for EVD, which had previously been relatively stalled in development despite the promising results in nonhuman primates, into accelerated production and clinical trials. Assuming that the findings of Ledgerwood et al. are confirmed, especially in African populations, cAd3EBO certainly warrants efficacy trials, but there are difficult decisions regarding the best dose and the trial design. In the current report, the authors have updated their findings, presenting evidence of continued immune humoral and CD4 and CD8 cellular immune responses at 48 weeks after vaccination, although the peak response was still at week 4 . Whether the immune response at 48 weeks is still sufficient to confer protection is unknown, given the previously mentioned lack of specific correlates of immunity. The authors note subsequently published results of phase 1 and 2 studies of a monovalent cAd3EBO vaccine, sometimes followed by an MVA boost, in which $1 \times 10^{11}$ particle units was determined to be the best dose on the basis of the immunogenicity and adverse event profile. Although use of the monovalent CAd3-EBO vaccine alone many be sufficient to confer short-term immunity, which is of clear value for outbreak control, the use of adjuvants may be needed for longer-term protection. Phase 3 trials are planned, but important questions remain: can traditional phase 2 and 3 efficacy trials be performed in West Africa, given the many ethical considerations; should the cAd3 and VSV vaccines be compared head-to-head; what should the target population be; and can it all be arranged in a human trial, or will we ultimately need to turn to the Food and Drug Administration Animal Rule (which stipulates that under certain circumstances, the FDA may grant approval on the basis of well-controlled studies in animals) $?^{10}$ The road is still long and there are many challenges, but we are nevertheless one step closer to a solution.

Disclosure forms provided by the author are available with the full text of this editorial at NEJM.org.

From the Tulane University Health Sciences Center, New Orleans; and the U.S. Naval Medical Research Unit No. 6, Lima, Peru.

A preliminary version of this editorial was published on November 26, 2014, at NEJM.org.

1. Nyenswah TG, Westercamp M, Kamali AA, et al. Evidence for declining numbers of Ebola cases - Montserrado County, Liberia, June-October 2014. MMWR Morb Mortal Wkly Rep 2014; 63:1072-6.

2. Ebola response roadmap situation report update. Geneva: World Health Organization, November 21, 2014 (http://apps.who .int/iris/bitstream/10665/144117/1/roadmapsitrep_21Nov2014_eng .pdf?ua=1).

3. Okware SI, Omaswa FG, Zaramba S, et al. An outbreak of Ebola in Uganda. Trop Med Int Health 2002;7:1068-75.

4. Ledgerwood JE, DeZure AD, Stanley DA, et al. Chimpanzee adenovirus vector ebola vaccine. N Engl J Med 2017;376:928-38.

5. Sullivan NJ, Martin JE, Graham BS, Nabel GJ. Correlates of protective immunity for Ebola vaccines: implications for regulatory approval by the animal rule. Nat Rev Microbiol 2009;7:393400.

6. Kugelman JR, Lee MS, Rossi CA, et al. Ebola virus genome plasticity as a marker of its passaging history: a comparison of in vitro passaging to non-human primate infection. PLoS One 2012;7(11):e50316.

7. Kimani D, Jagne YJ, Cox M, et al. Translating the immunogenicity of prime-boost immunization with ChAd63 and MVA ME-TRAP from malaria naive to malaria-endemic populations. Mol Ther 2014;22:1992-2003.

8. Stanley DA, Honko AN, Asiedu C, et al. Chimpanzee adenovirus vaccine generates acute and durable protective immunity against ebolavirus challenge. Nat Med 2014;20:1126-9.

9. Sheehy SH, Duncan CJ, Elias SC, et al. ChAd63-MVA-vectored blood-stage malaria vaccines targeting MSP1 and AMA1: assessment of efficacy against mosquito bite challenge in humans. Mol Ther 2012;20:2355-68.

10. Snoy PJ. Establishing efficacy of human products using animals: the US food and drug administration's "animal rule." Vet Pathol 2010;47:774-8.

DOI: 10.1056/NEJMe1414305

Copyright () 2017 Massachusetts Medical Society. 